\title{
Impact of COVID-19 infection on pregnant women: literature review
}

\author{
Ghadah Mohammed Alasbi ${ }^{1 *}$, Afaf Mohammad Saad ${ }^{2}$, Nada Jamal Abdulqader ${ }^{3}$, \\ Sulaiman Hemdi Laimooniah ${ }^{4}$, Fatimah Falah Aldajani ${ }^{5}$, Zaid Ali Althowimer ${ }^{6}$, \\ Ibrahim Sulaiman Alduraywish ${ }^{7}$, Atheer Saeed Al raqi ${ }^{8}$, Ahmed Ali Alahmari ${ }^{9}$, \\ Amjaad Fahad Algethami ${ }^{10}$, Atheer Saleh AlHumaid ${ }^{11}$, Ibtihaj Qaseem Alenezi ${ }^{12}$
}

\author{
${ }^{1}$ Department of Family Medicine, King Khalid University, Abha, Saudi Arabia \\ ${ }^{2}$ Department of Family Medicine, Aseer Central Hospital, Khamis Mushait, Saudi Arabia \\ ${ }^{3}$ College of Medicine, Ibn Sina National College, Jeddah, Saudi Arabia \\ ${ }^{4}$ College of Medicine, Umm Al-Qura University, Mecca, Saudi Arabia \\ ${ }^{5}$ Abqaiq PHCC, Ministry of Health, Abqaiq, Saudi Arabia \\ ${ }^{6}$ College of Medicine, Alfarabi Colleges, Riyadh, Saudi Arabia \\ ${ }^{7}$ General Physician, Domat Aljandal General Hospital, Aljouf, Saudi Arabia \\ ${ }^{8}$ College of Medicine, Taif University, Taif, Saudi Arabia \\ ${ }^{9}$ Department of Pharmacy, Medical Center Prince Naif Campus, Riyadh, Saudi Arabia \\ ${ }^{10}$ Department of Obstetrics and Gynecology, Maternity and Children Hospital, Mecca, Saudi Arabia \\ ${ }^{11}$ College of Medicine, Imam Muhammad Ibn Saud Islamic University, Riyadh, Saudi Arabia \\ ${ }^{12}$ Department of Pediatrics, Maternity and Children Hospital, Arar, Saudi Arabia
}

Received: 29 December 2020

Accepted: 05 January 2021

\section{*Correspondence: \\ Dr. Ghadah Mohammed Alasbi, \\ E-mail: dr.ghadah88@hotmail.com}

Copyright: (c) the author(s), publisher and licensee Medip Academy. This is an open-access article distributed under the terms of the Creative Commons Attribution Non-Commercial License, which permits unrestricted non-commercial use, distribution, and reproduction in any medium, provided the original work is properly cited.

\section{ABSTRACT}

SARS-CoV-2 infection in high-risk groups is associated with high mortality and morbidity. Pregnant females are considered one of these cases. More studies are needed to understand how the virus affects the complication rate in these cases and what is the best management plan. Hence, this study was conducted to stand on the real incidence and complications of COVID-19 in pregnancy. Databases like PubMed, Medline, Web of science, Embase, Google scholar, and Scopus were searched using the following search terms "severe acute respiratory syndrome of coronavirus-2" or "SARS-CoV-2" or "COVID-19" or "coronavirus" and "pregnant" or "neonate" or "pregnancy" transplacental transmission" or "placental transmission" or "vertical transmission" or intrauterine or perinatal. Pregnant women are not considered high-risk groups for COVID-19 infections as it was found that the risk of complication in COVID-19 patients was similar to non-infected patients. However, virulent strains of the virus had caused severe infections and complications. The study lacked a clear plan for the management of the virus in pregnant females.

Keywords: COVID-19, Infection, Pregnancy

\section{INTRODUCTION}

COVID-19 is considered one of the most infectious cases of pneumonia in recent days causing more than three million infections resulting in more than 240000 deaths. ${ }^{1}$
It is caused by Severe acute respiratory syndrome of coronavirus-2 (SARS-CoV-2) and it is transmitted through droplets to the mouth, nose, or eye. ${ }^{2}$ The disease usually resolves without treatment. However, complications occur in the elderly and patients with 
chronic conditions resulting in a high mortality rate in these populations. ${ }^{3}$

The disease usually passes unnoticed; however, in some cases, was associated with many complications. The disease needs between 5 to 14 days during which the patient is highly infective. The most common manifestations are fever, headache, fatigue, and myalgia. ${ }^{4}$ In young adults, the disease usually asymptomatic and pass without complications. However, it can develop complications in high-risk groups including young adults diagnosed with diabetes and hypertension. One of the least studied risk groups is pregnant female patients. ${ }^{5}$

Many studies reported the infection of pregnant females and both the maternal and neonatal outcomes. ${ }^{6,7}$ However, there is no worldwide protocol for the treatment of coronavirus in pregnant patients. The COVID-19 infection in pregnant women had a similar outcome as in non-infected females. ${ }^{8}$ The largest case series of 99 pregnant females reported that there were no complications in this cohort of pregnant females. ${ }^{9}$ In addition, no neonatal complications were reported, and the study found that there was no evidence of vertical transmission. ${ }^{9}$ Despite the results of this cohort, two case series reported maternal death after COVID-19 infection. ${ }^{10,11}$ It was also found the amniotic and placental tissues had positive SARS-CoV-2 antibodies implying vertical transmission. The neonatal outcome in these cases developed a fever and mild respiratory distress. The studies concluded that indicate a virulent strain of SARSCoV-2 can cause complications to both the mother and the fetus. ${ }^{10,11}$ However, most of the studies reported good outcomes associated with COVID-19 infections in pregnant women. ${ }^{9,12-17}$

Current study, is a review of maternal and neonatal complications after COVID-19 infection. Literature regarding the possible management of COVID-19 infection in pregnant females was also reviewed. A systematic search was conducted to identify relevant studies in the following databases: PubMed, Medline, Web of science, Embase, Google Scholar, and Scopus. The following search terms were used "severe acute respiratory syndrome of coronavirus-2" or "SARS-CoV2" or "COVID-19" or "coronavirus" and "pregnant" or "neonate" or "pregnancy" transplacental transmission" or "placental transmission" or "vertical transmission" or intrauterine or perinatal. The reference lists were manually searched to identify additional relevant studies meeting inclusion criteria. We included any study that reports the epidemiology, clinical characteristics, outcomes of neonatal and maternal infection of COVID19 in pregnant patients. No restrictions were applied.

\section{MATERNAL OUTCOMES}

Based on the literature, $95 \%$ of 275 pregnant females were tested positive for COVID-19 while the remaining women were diagnosed using external criteria and chest
X-ray. All studies with an incidence rate of more than $15 \%$ were notably from the United States except one study from France. It was also reported that one in 20 asymptomatic pregnant mothers were diagnosed as COVID-19. Chest X-ray had a ground-glass appearance and patches of pneumonia. The infected pregnant women's age ranged from 20 to 44 years and the gestational age on admission ranged from 5 to 41 weeks. ${ }^{5,14-16}$

There were four cases of miscarriage and abortion; over $50 \%$ of the cases gave birth with no complications while three chose to terminate their pregnancy. ${ }^{5,14-16}$ Of those who gave birth, $77 \%$ of cases delivered at term while only $23 \%$ had preterm pregnancy (Figure 1). The causes of preterm delivery in these cases were foetal distress, the start of antiviral treatment, and worsening of respiratory symptoms. $11 \%$ of pregnant women had emergency delivery. ${ }^{6,8,9}$ The indications for the emergency delivery are mainly for pure obstetric reasons like placenta previa, foetal distress, failed labor induction, and arrest of cervical dilatation. Other cases had emergency delivery after diagnosis of COVID-19 mainly due to respiratory complications and the mother needed anti-viral treatment. The most reported symptoms were fever, cough, myalgia, malaise, or fatigue..$^{9,15-17}$

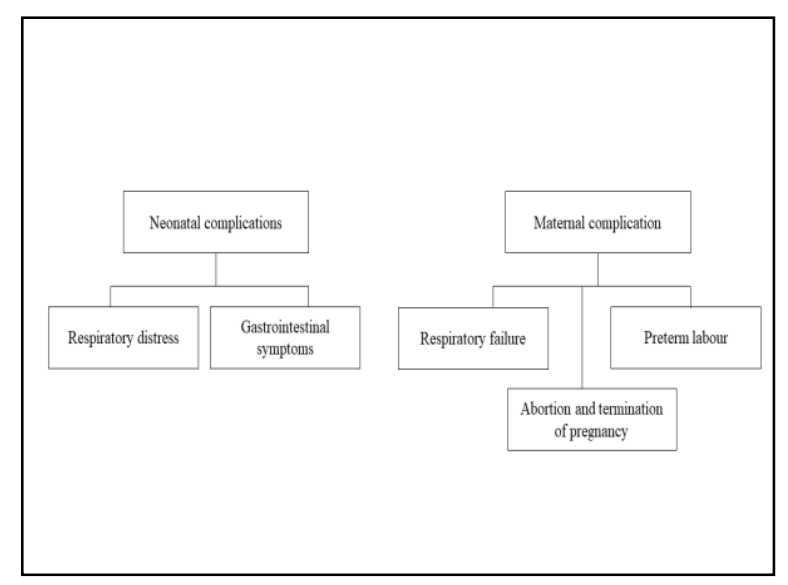

Figure 1: Maternal and neonatal complications.

Other rare symptoms were dyspnea, diarrhea, and abdominal pain; $8 \%$ of these cases were asymptomatic. Surprisingly, most of the cases started for a mean duration of 10 days before delivery. ${ }^{18-20}$ The remarkable lab findings were lymphopenia and lymphopenia, and positive IgG and IgM. Most of the cases had a typical chest CT scan of viral pneumonia. There was no specific treatment protocol used for all the pregnant patients as it was most dependent on the clinical condition of the patients and physician decision. ${ }^{9,12-15}$ Although most of the cases had less severe symptoms of COVID-19, complications were reported in a few cases. 36 pregnant women were admitted to the intensive care unit (ICU) and required oxygen therapy. The other five pregnant women had invasive ventilation and only one woman had

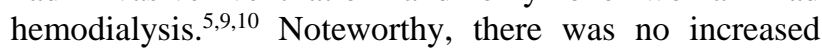


incidence of diabetes, hypertension, and pre-eclampsia in COVID-19 patients. $^{9}$

\section{NEONATAL OUTCOME}

Around $72 \%$ of neonates born to COVID-19 pregnant women were full-term with an average weight of about 2914 g. ${ }^{14}$ Only eight neonates weighted less than $2500 \mathrm{~g}$. In some studies, the neonates had throat/nasopharyngeal swab and serological IgG and IgM. 92\% of cases had negative results although some of the neonates developed fever, respiratory distress, and gastrointestinal symptoms (Figure 1). ${ }^{9}$ Another study reported complications in COVID-19 negative patients including thrombocytopenia, liver failure, pneumothorax, vomiting, and disseminated intravascular coagulation (DIC), however, these cases were preterm neonates. ${ }^{20}$ Another study reported cases of stillbirth born to mothers diagnosed with severe pneumonia. In total, sixteen neonates had tested positive for the COVID-19; nine of these cases were born to mothers diagnosed during pregnancy or after delivery by three days while the remaining neonates were born to non-tested mothers. 14 neonates had symptoms including fever, respiratory distress, and gastrointestinal symptoms, however, no deaths or complications were developed. ${ }^{11,14,18,21}$

All neonates except five neonates had leukocytosis, lymphocytopenia, and thrombocytopenia. All chest CTs revealed a typical picture of pneumonia. Some studies tested the possibility of vertical transmissions through testing the presence of the virus in amniotic fluid, cord blood, placenta, vaginal and cervical fluids, and no virus was found in these samples., ${ }^{4,22}$ It was also reported that there is a high incidence of neonatal intensive care admission in case of COVID-19 infected mothers..$^{23-25}$

However, two other studies found SARS-CoV-2 in the amniotic fluid, neonatal nasal, and throat swabs of two neonates born to mothers died due to COVID-19. ${ }^{11,18}$ It is explained in some studies that a pregnant mother may have virulent strain and the mothers contracted the virus preterm and died due to the infection. ${ }^{26-28}$ The virus can be transferred through vertical transmission through Angiotensin-converting enzyme 2 (ACE2) that is expressed in the human placenta. ${ }^{11,18}$

The virus spike glycoproteins are broken down by transmembrane protease serine 2 that increases the viral penetration and replication. ${ }^{29-34}$ It was found that transmembrane protease serine 2 is expressed in the placenta. ${ }^{14}$ Another possible hypothesis that infected women usually have a hypoxic placenta that impairs the placental barriers causing infectious agents to pass through the placenta to the infant. ${ }^{14-16}$

\section{RISK FACTORS FOR MORBIDITY AND MORTALITY}

History of chronic diseases was associated with worse outcomes in pregnant females. The most prevalent disease was diabetes mellitus. ${ }^{35-42}$ Gestational diabetes mellitus was also associated with a high risk of infection. Old maternal age was also associated with higher risk of admission compared to pregnant non-infected patients. ${ }^{43-}$ 50 In addition, chronic hypertension, high body mass index was associated with a high risk of admission and invasive ventilation. A high body mass index was correlated with the duration of invasive ventilation. Furthermore, recently pregnant females diagnosed with COVID-19 are usually more prone to abortion and complications. It was also reported that there is a high risk of complications if one of the family had confirmed COVID-19 infection. . $^{2,51,52}$

\section{Management of cases}

Most cases had antibiotic therapy and only $17.6 \%$ had received combined antiviral therapy and corticosteroids. ${ }^{18}$ Only two cases received hydroxychloroquine. ${ }^{11}$ In another study, the patients received a Chinese medication called lianhua qingwen. ${ }^{33,38,53-56}$ However, there was no protocol for the management of cases that require more trials and studies into this area. Most studies prevent breastfeeding till the mother becomes negative. However, this recommendation was not based on any evidence as it was reported that all breast milk showed negative results. ${ }^{5}$ A study reported that neonates who were breastfed by their mothers who tested positive for COVID-19 had favorable outcomes. ${ }^{34,57-60}$ It is more likely that delaying breastfeeding or mother neonates contact had worse outcomes than the risk of COVID-19. ${ }^{9}$ Meanwhile, other studies recommend that there is no urgent need for pregnancy termination, and it is more beneficial to deal with maternal pneumonia.

\section{CONCLUSION}

Based on the literature, COVID-19 in pregnancy did not increase the risk of neonatal and maternal complications. However, virulent strains of the virus can cause severe complications up to maternal and neonatal death. More studies are needed to agree on a protocol for the treatment of COVID-19 in these cases. Furthermore, more studies are needed to know whether the infected mother should breastfeed and have contact with her newborn or not.

\author{
Funding: No funding sources \\ Conflict of interest: None declared \\ Ethical approval: Not required
}




\section{REFERENCES}

1. Louapre C, Collongues N, Stankoff B. Clinical characteristics and outcomes in patients with coronavirus disease 2019 and multiple sclerosis. JAMA Neurol. 2020;77(9):1079-88.

2. Dawood FS, Ricks P, Njie GJ. Observations of the global epidemiology of COVID-19 from the prepandemic period using web-based surveillance: a cross-sectional analysis. Lancet Infect Dis. 2020;20 (11):1255-62.

3. Mao L, Jin H, Wang M, Hu Y, Chen S, He Q, Chang J, et al. Neurologic Manifestations of Hospitalized Patients With Coronavirus Disease 2019 in Wuhan, China. JAMA Neurol. 2020;77(6):683-90.

4. Wang W, Xu Y, Gao R, et al. Detection of SARSCoV-2 in different types of clinical specimens. JAMA. 2020;323(18):1843-4.

5. Ai T, Yang Z, Hou H. Correlation of chest CT and RT-PCR Testing for coronavirus disease 2019 (COVID-19) in China: A report of 1014 cases. Radiol. 2020;296(2):E32-40.

6. Alzamora MC, Paredes T, Caceres D, Webb CM, Valdez LM, La Rosa M. Severe COVID-19 during pregnancy and possible vertical transmission. Am J Perinatol. 2020;37(8):861-5.

7. Chawanpaiboon S, Vogel JP, Moller AB. Global, regional, and national estimates of levels of preterm birth in 2014: a systematic review and modelling analysis. Lancet Glob Health. 2019;7(1):e37-46.

8. Chen R, Zhang Y, Huang L, Cheng BH, Xia ZY, Meng QT. Safety and efficacy of different anesthetic regimens for parturients with COVID-19 undergoing Cesarean delivery: a case series of 17 patients. Can J Anaesth. 2020;67(6):655-63.

9. Ferrazzi E, Frigerio L, Savasi V, Vergani P, Prefumo $\mathrm{F}$, Barresi S, et al. Vaginal delivery in SARS-CoV-2infected pregnant women in Northern Italy: a retrospective analysis. BJOG. 2020;127(9):1116-21.

10. Alzamora MC, Paredes T, Caceres D, Webb CM, Valdez LM, La Rosa M. Severe COVID-19 during pregnancy and possible vertical transmission. Am J Perinatol. 2020;37(8):861-5.

11. Liu Y, Chen H, Tang K, Guo Y. Clinical manifestations and outcome of SARS-CoV-2 infection during pregnancy. J Infect. 2020;4453 (0120):3109.

12. Chen S, Huang B, Luo DJ. Pregnancy with new coronavirus infection: clinical characteristics and placental pathological analysis of three cases. China J Pathol. 2020;49(5):418-23.

13. Dong L, Tian J, He S, et al. Possible Vertical Transmission of SARS-CoV-2 From an Infected Mother to Her Newborn. JAMA. 2020;323(18):18468.

14. Gidlöf S, Savchenko J, Brune T, Josefsson $H$. COVID-19 in pregnancy with comorbidities: More liberal testing strategy is needed. Acta Obstet Gynecol Scand. 2020;99(7):948-9.
15. Han MS, Seong MW, Heo EY, Park JH, Kim N, Shin S, Cho SI, Park SS, Choi EH. Sequential analysis of viral load in a neonate and her mother infected with severe acute respiratory syndrome coronavirus 2 . Clin Infect Dis. 2020;71(16):2236-9.

16. Karimi-Zarchi M, Neamatzadeh H, Dastgheib SA. Vertical Transmission of coronavirus disease 19 (covid-19) from infected pregnant mothers to neonates: a review. Fetal Pediatr Pathol. 2020;39(3): 246-50.

17. Lee DH, Lee J, Kim E, Woo K, Park HY, An J. Emergency cesarean section performed in a patient with confirmed severe acute respiratory syndrome Coronavirus-2 -a case report. Korean J Anesthesiol. 2020;73(4):347-51.

18. Liu D, Li L, Wu X, Zheng D, Wang J, Yang L, Zheng C. Pregnancy and Perinatal Outcomes of Women With Coronavirus Disease (COVID-19) Pneumonia: A Preliminary Analysis. AJR Am J Roentgenol. 2020;215(1):127-32.

19. Parazzini F, Ferrazzi E, Ferrazzi E. Mode of delivery and clinical findings in COVID-19 infected pregnant women in. Lancet. 2020;20(10):458-65.

20. Paret M, Lighter J, Pellett Madan R, Raabe VN, Shust GF, Ratner AJ. Severe Acute Respiratory Syndrome Coronavirus 2 (SARS-CoV-2) Infection in Febrile Infants Without Respiratory Distress. Clin Infect Dis. 2020;71(16):2243-5.

21. Liu Y, Chen H, Tang K, Guo Y. Clinical manifestations and outcome of SARS-CoV-2 infection during pregnancy. J Infect. 2020.

22. Zeng $\mathrm{H}, \mathrm{Xu} \mathrm{C}$, Fan J. Antibodies in infants born to mothers with COVID-19 pneumonia. JAMA. 2020; 323(18):1848-9.

23. Chen D, Yang H, Cao Y, Cheng W, Duan T, Fan C, et al. Expert consensus for managing pregnant women and neonates born to mothers with suspected or confirmed novel coronavirus (COVID-19) infection. Int J Gynaecol Obstet. 2020;149(2):130-6.

24. Karimi-Zarchi M, Neamatzadeh H, Dastgheib SA. Vertical Transmission of Coronavirus Disease 19 (COVID-19) from Infected Pregnant Mothers to Neonates: A Review. Fetal Pediatr Pathol. 2020;39 (3):246-50.

25. Li N, Han L, Peng M, Lv Y, Ouyang Y, Liu K, et al. Maternal and neonatal outcomes of pregnant women with coronavirus disease 2019 (covid-19) pneumonia: a case-control study. Clin Infect Dis. 2020;71(16):2035-41.

26. Trippella G, Ciarcià M, Ferrari M, Buzzatti C, Maccora I, Azzari C, Dani C, Galli L, Chiappini E. COVID-19 in Pregnant women and neonates: A systematic review of the literature with quality assessment of the studies. Pathogens. 2020 ;9(6):485.

27. Wu YT, Liu J, Xu JJ. Neonatal outcome in 29 pregnant women with COVID-19: A retrospective study in Wuhan, China. PLoS Med. 2020;17(7): e1003195.

28. Yu N, Li W, Kang Q, Xiong Z, Wang S, Lin X, et al. Clinical features and obstetric and neonatal outcomes 
of pregnant patients with COVID-19 in Wuhan, China: a retrospective, single-centre, descriptive study. Lancet Infect Dis. 2020;20(5):559-64.

29. Mirzadeh M, Khedmat L. Pregnant women in the exposure to COVID-19 infection outbreak: the unseen risk factors and preventive healthcare patterns. J Matern Fetal Neonatal Med. 2020:1-2.

30. Qiao J. What are the risks of COVID-19 infection in pregnant women?. Lancet. 2020;395(10226):760-2.

31. Savasi VM, Parisi F, Patanè L, Ferrazzi E, Frigerio L, Pellegrino A, et al. Clinical findings and disease severity in hospitalized pregnant women with coronavirus disease 2019 (COVID-19). Obstet Gynecol. 2020;136(2):252-8.

32. Schmid MB, Fontijn J, Ochsenbein-Kölble N, Berger C, Bassler D. COVID-19 in pregnant women. Lancet Infect Dis. 2020;20(6):653.

33. Yan J, Guo J, Fan C, Juan J, Yu X, Li J, et al. Coronavirus disease 2019 in pregnant women: a report based on 116 cases. Am J Obstet Gynecol. 2020;223(1):111.e1-14.

34. Zhang L, Jiang Y, Wei M, Cheng BH, Zhou XC, Li $\mathrm{J}$, et al. Analysis of the pregnancy outcomes in pregnant women with COVID-19 in Hubei Province. Zhonghua Fu Chan Ke Za Zhi. 2020;55(3):166-71.

35. Afshar Y, Gaw SL, Flaherman VJ, Chambers BD, Krakow D, Berghella V, et al. Clinical Presentation of Coronavirus Disease 2019 (COVID-19) in Pregnant and Recently Pregnant People. Obstet Gynecol. 2020;136(6):1117-25.

36. Berhan Y. What immunological and hormonal protective factors lower the risk of COVID-19 related deaths in pregnant women?. J Reprod Immunol. 2020;142:103180.

37. Blauvelt CA, Chiu C, Donovan AL, Prahl M, Shimotake TK, George RB, et al. Acute Respiratory Distress Syndrome in a Preterm Pregnant Patient With Coronavirus Disease 2019 (COVID-19). Obstet Gynecol. 2020;136(1):46-51.

38. Breslin N, Baptiste C, Gyamfi-Bannerman C, Miller $\mathrm{R}$, Martinez R, Bernstein $\mathrm{K}$, et al. Coronavirus disease 2019 infection among asymptomatic and symptomatic pregnant women: two weeks of confirmed presentations to an affiliated pair of New York City hospitals. Am J Obstet Gynecol MFM. 2020;2(2):100118.

39. Chen H, Guo J, Wang C, Luo F, Yu X, Zhang W,et al. Clinical characteristics and intrauterine vertical transmission potential of COVID-19 infection in nine pregnant women: a retrospective review of medical records. Lancet. 2020;395(10226):809-15.

40. Chen L, Li Q, Zheng D, Jiang H, Wei Y, Zou L, et al. Clinical Characteristics of pregnant women with Covid-19 in Wuhan, China. N Engl J Med. 2020;382 (25):e100.

41. Delahoy MJ, Whitaker M, O'Halloran A, Chai SJ, Kirley PD, Alden N, et al. Characteristics and maternal and birth outcomes of hospitalized pregnant women with laboratory-confirmed COVID-19 COVID-NET, 13 States, March 1-August 22, 2020.
MMWR Morb Mortal Wkly Rep. 2020;69(38):13471354.

42. Dong H, Hu R, Lu C, Huang D, Cui D, Huang G, et al. Investigation on the mental health status of pregnant women in China during the Pandemic of COVID-19. Arch Gynecol Obstet. 2020:1-7.

43. Ferraiolo A, Barra F, Kratochwila C. Report of Positive Placental Swabs for SARS-CoV-2 in an Asymptomatic Pregnant Woman with COVID-19. Medicina (Kaunas). 2020;56(6):306.

44. Goldfarb IT, Clapp MA, Soffer MD, Shook LL, Rushfirth K, Edlow AG, et al. Prevalence and severity of coronavirus disease 2019 (covid-19) illness in symptomatic pregnant and postpartum women stratified by hispanic ethnicity. Obstet Gynecol. 2020;136(2):300-2.

45. Gonzalez-Brown VM, Reno J, Lortz H, Fiorini K, Costantine MM. Operating Room guide for confirmed or suspected COVID-19 pregnant patients requiring cesarean Delivery. Am J Perinatol. 2020; 37(8):825-8.

46. Heath PT, Le Doare K, Khalil A. Inclusion of pregnant women in COVID-19 vaccine development. Lancet Infect Dis. 2020;20(9):1007-8.

47. Hou L, Li M, Guo K, Wang W, Li B, Li J, et al. First successful treatment of a COVID-19 pregnant woman with severe ARDS by combining early mechanical ventilation and ECMO. Heart Lung. 2021;50(1):33-6.

48. $\mathrm{Hu}$ YJ, Wake M, Saffery R. Clarifying the sweeping consequences of covid-19 in pregnant women, newborns, and children with existing cohorts. JAMA Pediatr. 2020.

49. Jiao J. Under the epidemic situation of COVID-19, should special attention to pregnant women be given?. J Med Virol. 2020;92(9):1371-2.

50. Kajdy A, Feduniw S, Ajdacka U. Risk factors for anxiety and depression among pregnant women during the COVID-19 pandemic: A web-based crosssectional survey. Medicine (Baltimore). 2020;99(30): e21279.

51. London V, McLaren R Jr, Atallah F, Cepeda C, McCalla S, Fisher N, Stein JL, Haberman S, Minkoff H. The Relationship between Status at Presentation and Outcomes among Pregnant Women with COVID-19. Am J Perinatol. 2020;37(10):991-4.

52. McCartney SA, Kachikis A, Huebner EM, Walker CL, Chandrasekaran S, Adams Waldorf KM. Obesity as a contributor to immunopathology in pregnant and non-pregnant adults with COVID-19. Am J Reprod Immunol. 2020;84(5):e13320.

53. Buonsenso D, Raffaelli F, Tamburrini E, Biasucci DG, Salvi S, Smargiassi A, et al. Clinical role of lung ultrasound for diagnosis and monitoring of COVID19 pneumonia in pregnant women. Ultrasound Obstet Gynecol. 2020;56(1):106-9.

54. Ceulemans M, Hompes T, Foulon V. Mental health status of pregnant and breastfeeding women during the COVID-19 pandemic: A call for action. Int $\mathbf{J}$ Gynaecol Obstet. 2020;151(1):146-7. 
55. London V, McLaren R, Stein J, Atallah F, Fisher N, Haberman S, et al. Caring for pregnant patients with COVID-19: practical tips getting from policy to practice. Am J Perinatol. 2020;37(8):850-3.

56. Luo Y, Yin K. Management of pregnant women infected with COVID-19. Lancet Infect Dis. 2020;20 (5):513-4.

57. Zamaniyan M, Ebadi A, Aghajanpoor S, Rahmani Z, Haghshenas M, Azizi S. Preterm delivery, maternal death, and vertical transmission in a pregnant woman with COVID-19 infection. Prenat Diagn. 2020;40 (13):1759-61.

58. Zambrano LI, Fuentes-Barahona IC, Bejarano-Torres DA, Bustillo C, Gonzales G, Vallecillo-Chinchilla G, et al. A pregnant woman with COVID-19 in Central America. Travel Med Infect Dis. 2020;36:101639.

59. Zhang J, Zhang Y, Huo S. Emotional Eating in Pregnant Women during the COVID-19 Pandemic and Its Association with Dietary Intake and Gestational Weight Gain. Nutrients. 2020;12(8): 2250 .

60. Zhang Y, Ma ZF. Psychological responses and lifestyle changes among pregnant women with respect to the early stages of COVID-19 pandemic. Int J Soc Psychiatry. 2020;20764020952116.

Cite this article as: Alasbi GM, Saad AM, Abdulqader NJ, Laimooniah SH, Aldajani FF, Althowimer ZA, et al. Impact of COVID-19 infection on pregnant women: literature review. Int $\mathbf{J}$ Community Med Public Health 2021;8:927-32. 